\title{
Endoscopic dissection of refractory pancreatic duct stricture via accessory pancreatic duct approach for concurrent treatment of anomalous pancreaticobiliary junction in aging patients
}

\author{
This article was published in the following Dove Medical Press journal: \\ Clinical Interventions in Aging
}

\author{
Tao Wang ${ }^{1,2, *}$ \\ Dan-qing Liu' ${ }^{1,2, *}$ \\ Xu-dong Wen ${ }^{2, *}$ \\ Bing-yin Zhang ${ }^{1,2, *}$ \\ Wei-hui Liu'
}

'Department of Gastroenterology and Hepatology, Sichuan Academy of Medical Sciences \& Sichuan Provincial People's Hospital, Chengdu, Sichuan Province 6I0072, China; ${ }^{2}$ Department of Minimally Invasive Surgery, General Surgery Center of PLA, Chengdu Military General Hospital, Chengdu, Sichuan Province 610083, China

*These authors contributed equally to this work
Correspondence: Bing-yin Zhang General Surgery Center of PLA, Chengdu Military General Hospital, Chengdu, Sichuan Province 610083, China Email 439721905@qq.com

Wei-hui Liu

Department of Gastroenterology and Hepatology, Sichuan Academy of Medical Sciences \& Sichuan Provincial People's Hospital, Chengdu, Sichuan Province 610072, China

Email weihuiliu1984@163.com
Background: Although endoscopic management of pancreatic strictures by dilation and stenting is well established, some high-grade strictures are refractory to conventional methods. Here, we report a novel technique via accessory pancreatic duct (APD) approach to simultaneously release chronic pancreatitis-associated pancreatic stricture and correct anomalous pancreaticobiliary junction (APBJ). Due to APBJ and stricture of proximal main pancreatic duct, the APD turned out to be compensatory expansion. The stiff stenosis was dissected along the axial of APD using needle-knife electrocautery or holmium laser ablation, and then the supporting stent was placed into the pancreatic body duct. By doing so, the outflow channels of pancreatic and biliary ducts were exquisitely separated.

Patients and methods: Two patients aged 69 and 71 years underwent stricture dissection and stent insertion for fluent drainage of pancreatic juice. The postoperative course was marked by complete abdominal pain relief and normal blood amylase recovery. In the first patient, wireguided needle-knife electrocautery under fluoroscopic control was applied to release refractory stricture. The second patient was treated by SpyGlass pancreatoscopy-guided holmium laser ablation to lift pancreatic stricture.

Results: Plastic stents in APD were removed at 3 months after surgery, and magnetic resonance imaging at 6 months showed strictly normal aspect of the pancreatic duct.

Conclusion: Although both cases were successful without severe complications, we recommend this approach only for selected patients with short refractory pancreatic strictures due to chronic pancreatitis. In order to prevent severe complications (bleeding, perforation or pancreatitis), direct-view endoscopy-guided electrotomy needs to be developed.

Keywords: needle-knife electrocautery, holmium laser ablation, pancreatic duct stricture, anomalous pancreaticobiliary junction (APBJ), endoscopic retrograde cholangiopancreatography (ERCP)

\section{Introduction}

The endoscopic techniques such as endoscopic retrograde cholangiopancreatography (ERCP), endoscopic sphincterotomy and endoscopic pancreatic stenting (EPS) are widely accepted as alternatives to surgery for the treatment of benign strictures in the main pancreatic duct (MPD). ${ }^{1}$ EPS has been proven effective to treat MPD stricture in chronic pancreatitis (CP), ${ }^{2}$ especially with peculiar stents such as fully covered self-expandable metal stent (FCSEMS) ${ }^{3,4}$ and s-type stent. ${ }^{5}$ To facilitate EPS, pancreatoscopy-guided cannulation, ${ }^{6}$ dilation with a controlled radial expansion 
balloon, ${ }^{7}$ wire-guided diathermic dilator ${ }^{8}$ or a Soehendra stent retriever, ${ }^{9,10}$ and a guidewire left in place for 24 hours ${ }^{11}$ have been reported to play a supporting role. However, there is no more frustrating time than when the stricture of the pancreatic duct is very severe, and only the guidewire can pass across it and there is no way to push any catheter or dilating device beyond the stricture. ${ }^{12}$ Under such circumstances, dissection of stricture with wire-guided needle-knife electrocautery may be a beneficial strategy. Although the endoscopic wire-guided needle-knife electrotomy is reported to be safe and effective in the treatment of refractory biliary stricture, ${ }^{13}$ there is little experience of its application in management of refractory MPD strictures, ${ }^{12,14}$ especially in those tubular strictures far from the opening of both MPD and accessory pancreatic duct (APD). In addition, if the stricture is extremely tight and tortuous such that even the guidewire is difficult to advance, then, needle-knife electrotomy cannot be safely implemented. While facing such a dilemma, SpyGlass pancreatoscopy-guided holmium laser ablation may serve as a complementary strategy. Here we describe two cases of successful endoscopic pancreatic duct incision for difficult MPD strictures via wire-guided needle-knife electrocautery or SpyGlass pancreatoscopy-guided holmium laser ablation. Although both cases achieved favorable results, we do not advocate this method for routine use because of the potential for severe complications, for example, bleeding, ductal perforation or pancreatic parenchymal damage. Written informed consents were provided by both patients to have the case details and any accompanying images published. Both surgical treatments and case details were discussed and approved by Sichuan Academy of Medical Sciences \& Sichuan Provincial People's Hospital and Chengdu Military Hospital Ethics Committee. The Declaration of Helsinki for Medical Research has been strictly adhered to.

\section{Case I: endoscopic wire-guided needle-knife electrocautery of pancreatic neck duct stricture via APD approach}

A 69-year-old woman with CP first diagnosed in 2006 was treated with expectant therapy and the patient achieved symptomatic relief. She was diagnosed with gall bladder stones previously in 2003, for which she underwent open cholecystectomy and bile duct exploration and attained complete remission. She developed dilation of both extrahepatic bile duct ( $2.0 \mathrm{~cm}$ in diameter) and MPD ( $0.6 \mathrm{~cm}$ in diameter), suffered from abdominal pain, and required endoscopic nasobiliary drainage or endoscopic nasopancreatic drainage every year since 2010. In 2013, imaging studies revealed evidence of pancreatic stricture combined with anomalous pancreaticobiliary junction (APBJ). Because the patient refused surgery to correct pathologic drainage of bile and pancreatic juice, minimally invasive dilation of the stricture under ERCP was performed in 2013, 2015 and 2016, respectively. Unfortunately, these resulted in limited improvement in both the degree of stenosis and the patient's symptoms. In 2017, the patient was presented to us with unrelenting pain following an episode of acute pancreatitis. In order to rectify stricture and release symptoms, it was decided to incise the fibrotic circle of pancreatic neck duct with endoscopic wire-guided needle-knife electrocautery technique.

Under duodenoscope, we noticed that the major duodenal papilla was situated between two diverticula (Figure 1A). Selective MPD cannulation was made using a precut sphincterotomy technique plus guidewire (Figure 1B). Figure 1C indicated that a $10 \mathrm{Fr}$ plastic stent was put into $\mathrm{CBD}$ to drainage bile,while Figure 1D showed that the cannulation was made in minor duodenal papilla. The water balloon dilation was exerted to make room for stent placement (Figure 1E). A 8.5Fr plastic stent was introduced to support dilated pancreatic duct (Figure 1F). After deep cannulation, the 0.035-inch guidewire (Jagwire/Zebra; Boston Scientific Corp., Natick, MA, USA) traversed through APD and ran into duodenum instead of advancing to the pancreatic body/ tail duct (Figure 2A). Pancreatography presented development of both pancreatic duct and bile duct, indicating APBJ (B-P type; Figure 2A). After several attempts, we made the guidewire to pass through APD and get to dilated pancreatic body/tail duct (1.2 cm in diameter). Pancreatography through APD ( $0.4 \mathrm{~cm}$ in diameter) showed a dominant stricture in the pancreatic neck duct (Figure 2B). The stenosis was so narrow that only the guidewire could pass through and lead to pancreatic body/tail duct. The conventional dilation of the stricture was attempted with a 6Fr catheter dilator (Wilson Cook Medical) or a 7Fr Soehendra stent retriever, and both failed. Intraluminal incision technique with needle-knife (MicroKnife XL sphincter tome; Boston Scientific Corp.) was applied. As a triple-lumen catheter tapered from 2.3 to $1.8 \mathrm{~mm}$ over the distal part, the needle-knife accommodated a 0.035-inch guidewire in one channel. The cutting wire was extruded $2 \mathrm{~mm}$, and electrocautery was applied on the stenosis via an electrosurgical generator (ARCO 2000; Söring Medizintechnik GmbH, Quickborn, Germany) until the knife passed through the stricture (Figure 2C). As the needle-knife advanced over the guidewire, the accessory catheter followed steadily to prevent extending the cutting wire out of pancreatic duct. Further dilation was then introduced by using a columnar 

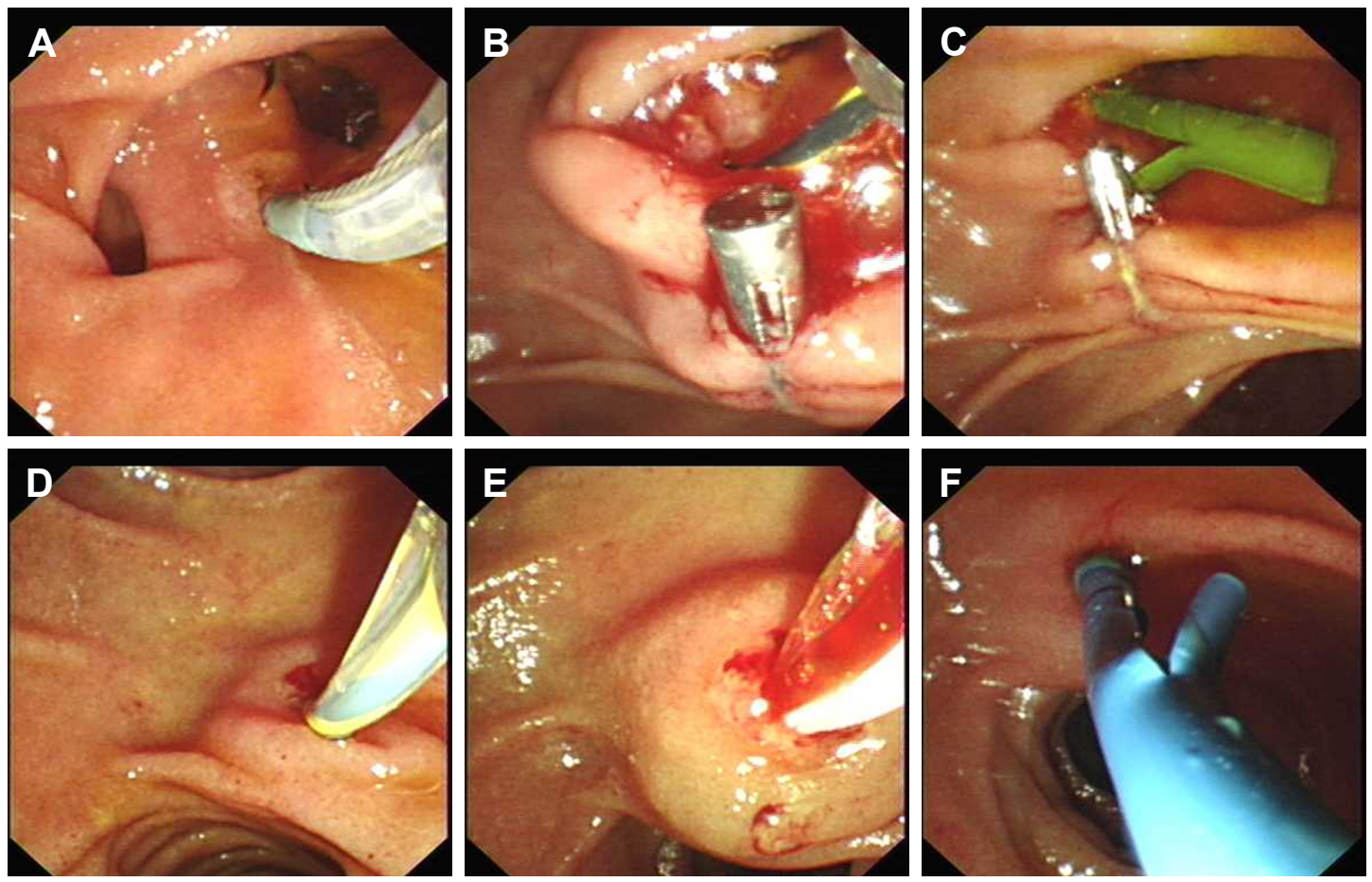

Figure I Endoscopic images showing treatment of pancreatic duct stricture and APBJ.

Notes: The major papilla was located in the middle of two diverticula (A). Precut of major duodenal papilla was smoothly operated (B). A I0Fr plastic stent was put into CBD to drain bile (C). Cannulation was made in minor duodenal papilla (D). Water balloon dilation was performed to make room for stent placement (E). An 8.5Fr plastic stent was introduced to support dilated pancreatic duct (F).

Abbreviations: $A P B J$, anomalous pancreaticobiliary junction; $C B D$, common bile duct.
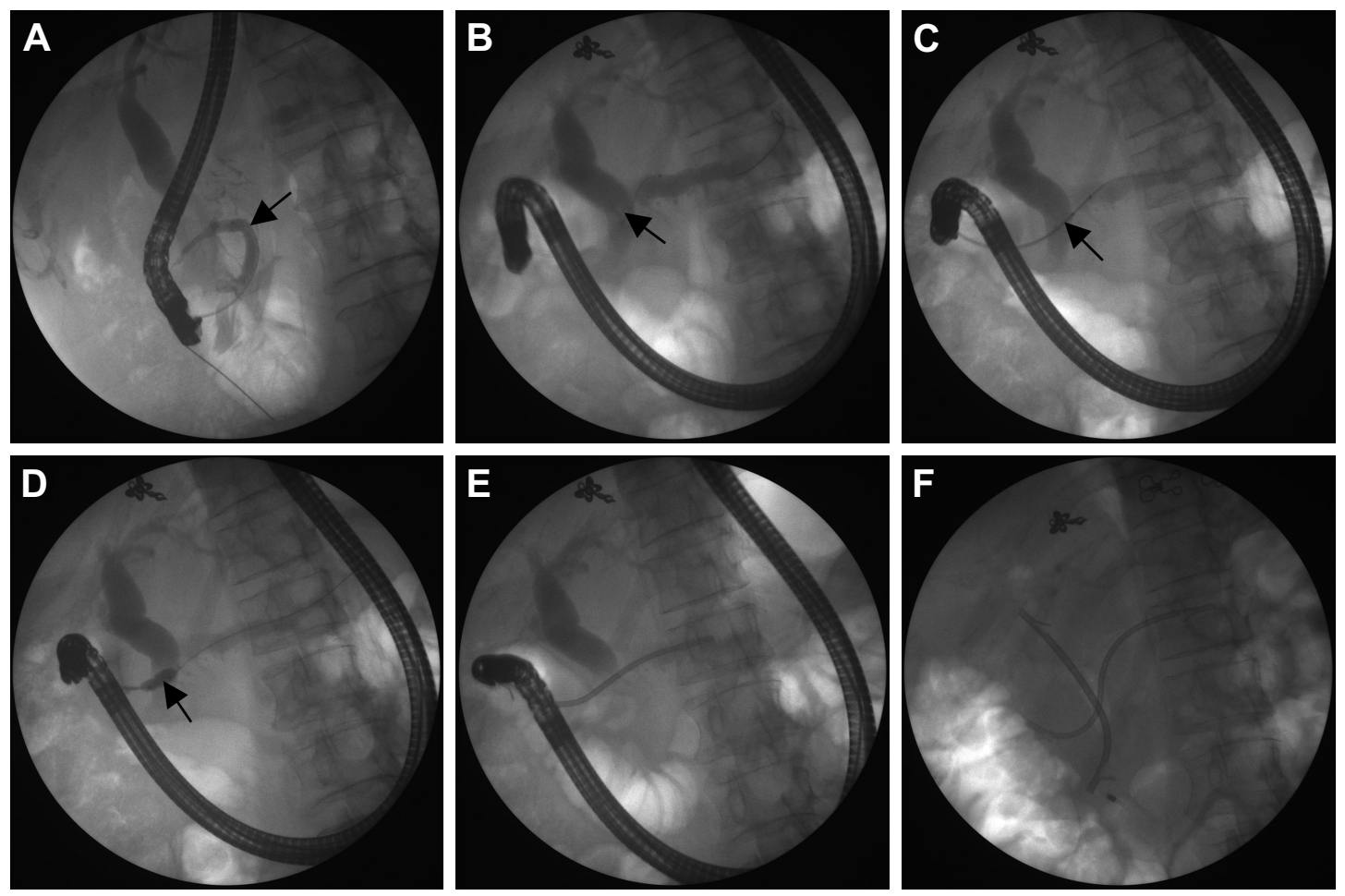

Figure 2 ERCP presenting diagnosis and therapy of pancreatic duct stricture and APBJ.

Notes: Pancreatography through MPD reveals APBJ (B-P type) (A). The arrow in (A) indicates the confluence of MPD and minor pancreatic duct. Pancreatography through APD shows a dominant stricture in the pancreatic neck duct (B). The arrow in (B) indicates the stenotic site. The needle-knife with electrocautery was successfully progressed over a guidewire after incising the refractory stricture in the blend current mode $(\mathbf{C})$. The arrow in (C) indicates the incised site. The stenotic pancreatic duct was further dilated using columnar water balloon (D). The arrow in (D) indicates the dilating site. An 8.5Fr plastic stent was placed into APD (E). A I0Fr plastic stent was implanted into CBD (F).

Abbreviations: APBJ, anomalous pancreaticobiliary junction; $C B D$, common bile duct; $E R C P$, endoscopic retrograde cholangiopancreatography; MPD, main pancreatic duct. 
water balloon (Figure 2D), so as to make the dilated duct capacious enough $(0.5 \mathrm{~cm}$ in diameter) for subsequent placement of 8.5Fr plastic stent (Figure 2E). After releasing the stricture of pancreatic duct, selective cannulation of common bile duct (CBD) was made. Finally, two stents were separately located in the bile duct and pancreatic duct to correct maljunction of pancreatic and biliary ducts (Figure 2F). At follow-up X-ray and magnetic resonance cholangiopancreatography 3 months later, no migration of inserted stents was observed and the dilated pancreatic neck duct was still fluent. During 4 months of follow-up, the patient has remained symptom-free and has normal liver function. Six months after treatment, the plastic stents will be moved according to therapeutic guideline. ${ }^{15}$ If necessary, FCSEMS can be placed into dilated pancreatic neck duct. ${ }^{16}$

\section{Case 2: SpyGlass pancreatoscopy- guided holmium laser ablation of pancreatic body duct stricture through APD access}

A 71-year-old female with a long history of CP was admitted to our hospital because of severe epigastric discomfort.
During ERCP, the cannulation of CBD was successfully performed; however, we failed to selectively insert guidewire into MPD after several attempts. Under fluoroscopic guidance (Figure 3A), SpyGlass cholangioscopy (Boston Scientific Corp.) was conducted and it revealed normal structure of bile ducts (Figure 3B). When scanning the CBD, the SpyGlass cholangioscopy was specially used to investigate the orifice of MPD. As the SpyGlass cholangioscopy was pulled back close to the distal CBD (Figure 3C), the opening of MPD was confirmed to have stiff stricture, which did not allow a 0.035-inch guidewire to pass through (Figure 3D). The SpyGlass cholangioscopy picture indicated APBJ with MPD joining CBD (P-B type). With successful cannulation of minor papilla, the pancreatography revealed stenosis in pancreatic body duct and dilatation of most pancreatic duct (Figure 3E). When the SpyGlass pancreatoscope was inserted and advanced to the pancreatic body duct, the extremely strictured site was clearly observed (Figure 3F). Under direct view guidance of SpyGlass pancreatoscopy (Figure 4A), a standard 0.025-inch guidewire (VisiGlide; Olympus Medical Systems, Tokyo, Japan) was incapable of advancing across the stricture in pancreatic body duct with repeated attempts (Figure 4B).
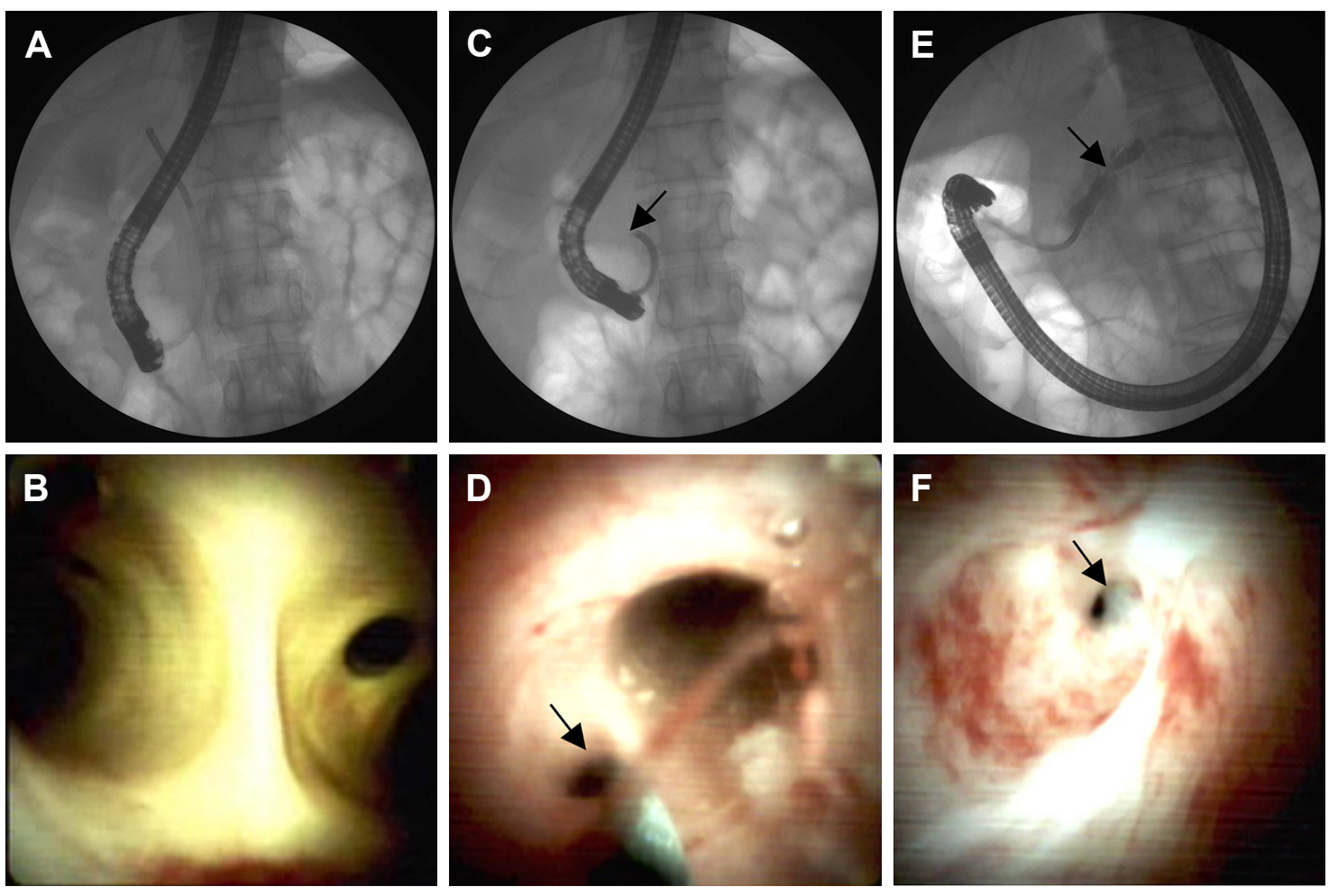

Figure 3 Endoscopic diagnosis of pancreatic duct stricture and APBJ.

Notes: Initial ERCP image following cannulation of major papilla revealed SpyGlass cholangioscopy entering CBD (A). The SpyGlass cholangioscopy image presented normal structures of both left and right bile ducts (B). As the SpyGlass cholangioscopy retreated along CBD (C). The arrow in (C) indicates the head of Spyglass cholangioscopy. The opening of MPD was detected joining distal CBD, suggesting possible APBJ (P-B type). (D). The arrow in (D) indicates the opening of MPD ERCP image with cannulation of minor papilla indicated a dilated and beaded pancreatic duct with stricture in pancreatic body duct (E). The SpyGlass pancreatoscopy study demonstrated extremely stenotic ring in pancreatic body duct (F). The arrow in (E) and (F) indicate the stenotic site.

Abbreviations: APBJ, anomalous pancreaticobiliary junction; CBD, common bile duct; ERCP, endoscopic retrograde cholangiopancreatography; MPD, main pancreatic duct. 

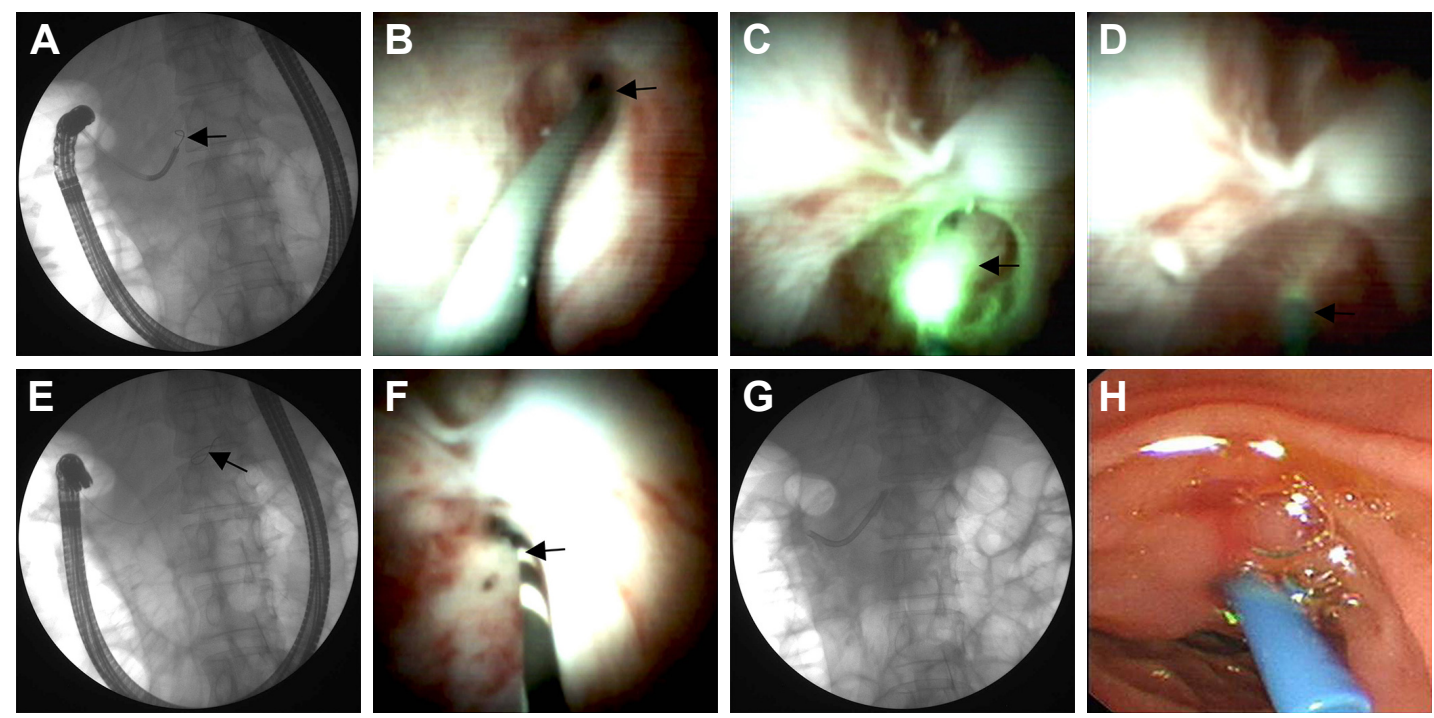

Figure 4 Endoscopic treatment of pancreatic duct stricture and APB].

Notes: Both ERCP image (A) and SpyGlass pancreatoscopy picture (B) showed guidewire failing to pass through contracted pancreatic body duct. SpyGlass pancreatoscopyguided holmium laser ablation of stricture was safely conducted $(\mathbf{C})$ with the laser probe traversing the stricture area (D). During pancreatography (E) and direct view of SpyGlass pancreatoscopy (F), the guidewire finally advanced across the narrowed duct of pancreatic body. After dilation of the pancreatic duct by a water balloon, a 7Fr plastic stent was implanted into the pancreatic duct via minor papilla $(\mathbf{G}, \mathbf{H})$. The arrow in $\mathbf{A}, \mathbf{B}, \mathbf{E}$, and $\mathbf{F}$ indicate the guide wires; the arrow in $\mathbf{C}$ and $\mathbf{D}$ indicate the laser probes. Abbreviations: APBJ, anomalous pancreaticobiliary junction; ERCP, endoscopic retrograde cholangiopancreatography.

Although we thought about using other dilating instruments such as Soehendra stent retrievers, it was not available without guidewire direction at the time. Considering a large quantity of contrast injected and the guidewire irritating frequently, we had to complete the pancreatic duct drainage in order to prevent aggravation of pancreatitis or infection in the pancreatic duct. As the stenotic site was about $5 \mathrm{~cm}$ from the orifice of APD, we judged that the distance was not short enough to control a needle-knife without guidewire navigation. Therefore, we decided to dissect the stricture by using SpyGlass pancreatoscopy-guided holmium laser ablation with a $365 \mathrm{~mm}$ SlimLine disposable laser probe (Lumenis, Santa Clara, CA, USA). With holmium laser precisely targeting the stenotic site of the pancreatic duct (Figure 4C), the stricture ring was safely incised open in a few seconds and the laser probe could easily pass through (Figure 4D). The patient reported little pain during dissection, and bleeding from the minor papilla after dissection was minor. Under fluoroscopy and SpyGlass pancreatoscopy, the guidewire could smoothly advance through primary contracted duct to reach pancreatic tail (Figure 4E and F). We could then deploy a 7Fr blue pancreatic stent (Advanix ${ }^{\circledR}$; Boston Scientific Corp.) into the APD (Figure 4G and $\mathrm{H}$ ). In this way, the pancreatic juice directly drained into the duodenum instead of flowing through distal CBD. One month later, an $8.5 \mathrm{Fr}$ pancreatic stent was inserted with no difficulty, and 3 months later, the pancreatic stent could be removed successfully without stenosis at the dissected area.

\section{Discussion}

An improvement of symptoms from a stricture of the pancreatic duct caused by $\mathrm{CP}$ and other benign pancreatic diseases can often be achieved with endoscopic pancreatic stent placement. ${ }^{15}$ However, the refractory stricture of a pancreatic duct far from the papilla sometimes makes this procedure difficult. In such circumstances, a Soehendra stent extractor can be used as a drill to make room for later stent implantation. ${ }^{17}$ Nevertheless, this procedure may induce irreversible changes in the pancreatic duct because of duct squash. In addition, as this technique is guidewire-dependent, if the guidewire is unable to pass through the pancreatic duct stricture, the Soehendra stent extractor has little chance to acquire success. Therefore, it is necessary to establish an alternative procedure for dissecting the pancreatic duct with or without a needle-knife. There is no doubt that cutting the tissue visibly under SpyGlass pancreatoscopy is safer and more efficient, while in practice, needle-knife is unable to go through the operation whole of SpyGlass pancreatoscopy.

Although pancreatic duct stricture can be modified by the above-mentioned endoscopic methods, when it combines with other anatomic abnormalities, such as APBJ, the treatment becomes a dilemma. In APBJ cases, early activation of pancreatin would lead to repeated episodes of pancreatitis or biliary carcinogenesis. ${ }^{18}$ Treatment of APBJ is still controversial. ${ }^{19}$ As the development of endoscopic strategy goes abroad, the pancreatic duct stricture and APBJ can be cured simultaneously. All kinds of pancreato-biliary disease 
can be managed by the endoscopic strategies. ${ }^{20}$ Here, we reported two cases of interest as they provide a minimally invasive therapeutic strategy for refractory pancreatic duct stricture associated with APBJ, which occurs at a large distance from the duodenal papilla. In these cases, due to APBJ and stricture in MPD, the APD is always compensatory expansion, which provides an access for endoscopic management. The endoscopic dissection technique via APD by using wire-guided needle-knife electrocautery or SpyGlass pancreatoscopy-guided holmium laser ablation could serve as not only an effective bridge treatment when surgery is not applicable, but also an ultimate treatment while other therapeutic methods fail. The first patient was treated by wire-guided needle-knife electrocautery and stent placement, which showed stiff stricture in the pancreatic neck duct and APBJ with CBD joining MPD. For the second case, as the refractory stenosis of the pancreatic body duct did not allow the guidewire to cross and the APBJ was presented by a $2 \mathrm{~cm}$ long common channel with the MPD filling from the CBD, the patient was treated with SpyGlass pancreatoscopyguided holmium laser ablation and stent implantation. These cases confirm that minimally invasive endoscopic approach is possible, safe and effective in simultaneously releasing pancreatic duct stricture and correcting APBJ with favorable results.

Although endoscopic dissection of pancreatic duct stricture and later implantation of plastic stent for pancreatic disorder is effective and convenient, this technique has its own limitations. On the one hand, the success of this technique is highly dependent on proficient skill and rich hands-on experience. On the other hand, this technique is almost in blind mode. Therefore, we do not advocate this procedure for routine use, because this maneuver has potential significant complications such as bleeding or perforation. ${ }^{21}$ The more distant the stenosis is located from the papilla, the more difficult the control of safe stricture dissection becomes. ${ }^{12}$ Fortunately, these complications did not occur in these patients. As a potential solution for preventing severe complications, we propose the use of a wire-guided needle-knife electrocautery under double direction of fluoroscopy and pancreatoscopy. Although this strategy was not used in this case, it is worth trying by using the combination of a 0.035 -inch guidewire, a KD441Q needle-knife (Olympus Medical Systems), ERCP and peroral pancreatoscopy. In addition, to achieve persistent improvement, it is recommended to replace the plastic stent with the FCSEMS for long-term support. The FCSEMS placement appears to be safe and effective for the treatment of benign refractory pancreatic ductal strictures with a long-term efficiency, ${ }^{4}$ especially in APBJ needing sufficient shunt of bile and pancreatic juice.

\section{Conclusion}

Currently, it is very tricky to deal with refractory strictures in pancreatic ducts, especially those strictures located far from the orifice of pancreatic duct. The technique for endoscopic dissection of stiff stenosis in pancreatic duct via APD approach can provide acceptable outcomes in patients with benign pancreatic disease. In addition, the implantation of stents via APD can separately drain the pancreatic juice into the duodenum, so as to correct APBJ. Although further study is still required for safety concerns, this technique may have potential for selected patients with refractory MPD strictures and APBJ.

\section{Acknowledgments}

The authors gratefully thank Drs Le Xiao and Hong-tao Yan for their great help in patient management.

This study was funded by Sichuan Science and Technology Project (No 18YYJC0442 and 2015JY0161) and Chinese National Natural Science Foundation (No 81502696).

\section{Disclosure}

All authors disclose no financial relationships relevant to this publication. The authors report no other conflicts of interest in this work.

\section{References}

1. Zheng MW, Qin MF, Cai W. Endoscopic pancreatic duct and biliary duct stenting in treatment of chronic pancreatitis with distal benign biliary stricture: a single-center experience. Hepatobiliary Pancreat Dis Int. 2011;10(5):539-543.

2. Seza K, Yamaguchi T, Ishihara T, et al. A long-term controlled trial of endoscopic pancreatic stenting for treatment of main pancreatic duct stricture in chronic pancreatitis. Hepatogastroenterology. 2011; 58(112):2128-2131.

3. Ang T. Chronic pancreatitis with pancreatic duct stricture and calculi treated by fully covered self-expandable metal stent placement and intraductal pancreatoscopy-guided laser lithotripsy. Endoscopy. 2017;49(06): E145-E146.

4. Oh D, Lee JH, Song TJ, et al. Long-term outcomes of 6-mm diameter fully covered self-expandable metal stents in benign refractory pancreatic ductal stricture. Dig Endosc. 2018;30(4):508-515.

5. Eickhoff A, Weickert U, Riemann JF. Efficacy of S-type stents for the treatment of the main pancreatic duct stricture in patients with chronic pancreatitis. Scand J Gastroenterol. 2007;42(4):537.

6. Chahal P, Topazian MD. Pancreatoscopy-guided cannulation of a difficult pancreatic stricture (with video). Gastrointest Endosc. 2009;69(7): 1385-1386; discussion 6-7.

7. Viazis N, Stefanidis G, Katsikani A, Pleskow D, Chuttani R. Pancreatic duct stricture dilation with a controlled radial expansion balloon for the management of stone impaction. Endoscopy. 2009;41(Suppl 2):E106-E107.

8. Kawakami H, Kuwatani M, Kawakubo K, et al. Transpapillary dilation of refractory severe biliary stricture or main pancreatic duct by using a wire-guided diathermic dilator (with video). Gastrointest Endosc. 2014;79(2):338-343. 
9. Ziebert JJ, Disario JA. Dilation of refractory pancreatic duct strictures: the turn of the screw. Gastrointest Endosc. 1999;49(5):632-635.

10. Brand B, Thonke F, Obytz S, et al. Stent retriever for dilation of pancreatic and bile duct strictures. brand@uke.uni-hamburg.de.Endoscopy. 1999;31(2):142-145.

11. Familiari P, Spada C, Costamagna G. Dilation of a severe pancreatic stricture by using a guidewire left in place for 24 hours. Gastrointest Endosc. 2007;66(3):618-620.

12. Kawamoto H, Ishida E, Ogawa $\mathrm{T}$, et al. Dissection of a refractory pancreatic-duct stricture by using a pre-cutting needle-knife. Gastrointest Endosc. 2006;63(1):190-192.

13. Yang YL, Zhang C, Zhao G, et al. Choledochoscopic high-frequency needle-knife electrotomy as an effective treatment for intrahepatic biliary strictures. J Gastroenterol Hepatol. 2015;30(9):1438-1443.

14. Gao DJ, Hu B, Pan YM, et al. Feasibility of using wire-guided needleknife electrocautery for refractory biliary and pancreatic strictures. Gastrointest Endosc. 2013;77(5):752-758.

15. Ogura T, Kurisu Y, Yamamoto K, et al. Placement of a novel fully covered metallic stent for refractory pancreatic duct stricture. Endoscopy. 2015;47 Suppl 1 UCTN(S 01):E206-E207.
16. Ogura T, Onda S, Takagi W, et al. Placement of a $6 \mathrm{MM}$, fully covered metal stent for main pancreatic head duct stricture due to chronic pancreatitis: a pilot study (with video). Therap Adv Gastroenterol. 2016;9(5): 722-728.

17. Yamaguchi $\mathrm{T}$, Ishihara $\mathrm{T}$, Tadenuma $\mathrm{H}$, et al. Use of a Soehendra stent retriever to treat a pancreatic pseudocyst with EUS-guided cystogastrostomy. Endoscopy. 2004;36(8):755.

18. Zimmer V, Heinrich C. West meets East: dynamic ERCP characterization of pancreaticobiliary maljunction without biliary dilation underlying extensive biliary tract cancer. Endoscopy. 2017;49(07):E175-E176.

19. Kamisawa T, Kuruma S, Tabata T, et al. Pancreaticobiliary maljunction and biliary cancer. $J$ Gastroenterol. 2015;50(3):273-279.

20. Beyna T, Neuhaus H, Gerges C. Endoscopic treatment of pancreatic duct stones under direct vision: revolution or resignation? Systematic review. Dig Endosc. 2018;30(1):29-37.

21. Costamagna G, Boškoski I. Needle-knife electrocautery: "give me a lever and I will move the world”. Gastrointest Endosc. 2013;77(5):759-760.
Clinical Interventions in Aging

\section{Publish your work in this journal}

Clinical Interventions in Aging is an international, peer-reviewed journal focusing on evidence-based reports on the value or lack thereof of treatments intended to prevent or delay the onset of maladaptive correlates of aging in human beings. This journal is indexed on PubMed Central, MedLine,

\section{Dovepress}

CAS, Scopus and the Elsevier Bibliographic databases. The manuscript management system is completely online and includes a very quick and fair peer-review system, which is all easy to use. Visit http://www.dovepress. com/testimonials.php to read real quotes from published authors. 